\title{
The beams with composite reinforcement inclined sections' finite element modeling operation results comparison with the results of the laboratory samples' tests
}

\author{
Alexander Shilov* and Petr Shilov \\ Don State Technical University, 344022, Rostov-on-Don, Russia
}

\begin{abstract}
The results of a numerical experiment are presented, within the framework of which finite element modeling of the reinforced concrete beams support sections with external composite reinforcement beams inclined sections operation was carried out, as well as their comparison with the results of an experiment carried out on real laboratory samples, similarly reinforced on the support areas by external composite U-shaped cross clamps. The strength of inclined sections of both real laboratory specimens and their design models was considered. Both numerical and real experiments were carried out at three values of the shear fracture span: $1.5 h_{0} ; 2.0 h_{0}$ and $2.5 h_{0}$. The obtained results of the numerical experiment are compared with the results of laboratory tests on beams in order to analyze the accuracy of the calculation results and to find the optimal method for modeling and taking into account the external composite reinforcement clamps in the design models that would most accurately reflect their contribution to the operation of the inclined section of bent reinforced concrete elements. For the numerical experiment, the method of finite element modeling of samples was used. An assessment of the method used for computational modeling of the operation of inclined sections reinforced with external composite reinforcement is given.
\end{abstract}

\section{Introduction}

The Building Code publication on the reinforcement of reinforced concrete structures with composite materials revealed many points of interest for scientific research in Russia. This interest is largely due to the fact that, many issues related to the operation of a reinforced concrete element reinforced with composite materials were not covered in the mentioned regulatory document.

As part of a large-scale study of the work of reinforced concrete structures reinforced with composite materials, at the Department of Reinforced Concrete and Stone Structures, DSTU, the operation issues of reinforced concrete structures reinforced with round glass and carbon fiber reinforcement [1-4], the sections strength of reinforced concrete beams normal to the longitudinal axis of the element, reinforced external composite reinforcement

*Corresponding author: ale381420082008@yandex.ru 
[5], compressed reinforced concrete elements reinforced with composite materials at different eccentricities of load application [6-9]. Also, a study on the sections of reinforced concrete beams that are inclined to the longitudinal axis of the element, reinforced with external composite clamps in the presence and absence of initial inclined cracks was carried out [10-14].

To confirm the reliability of the experimental data obtained during the reinforced inclined sections strength study, finite element modeling was performed in the LIRA-SAPR 2013 software package. In order to save computer time, a quarter of the structure was considered. Concrete was modeled by volumetric physically nonlinear finite elements in the form of parallelepipeds (FE 231, Fig. 1). Minimum FE side size was $0.0125 \mathrm{~m}$. For steel longitudinal and transverse reinforcement, universal physically nonlinear spatial bar finite elements were used (FE 210, Fig. 2), and composite clamps were modeled as physically nonlinear universal rectangular FE shells (FE 241, Fig.3). The design scheme for the span of the shear fracture $\mathrm{a}=0.33 \mathrm{~m}$ is shown in Fig. 4 .

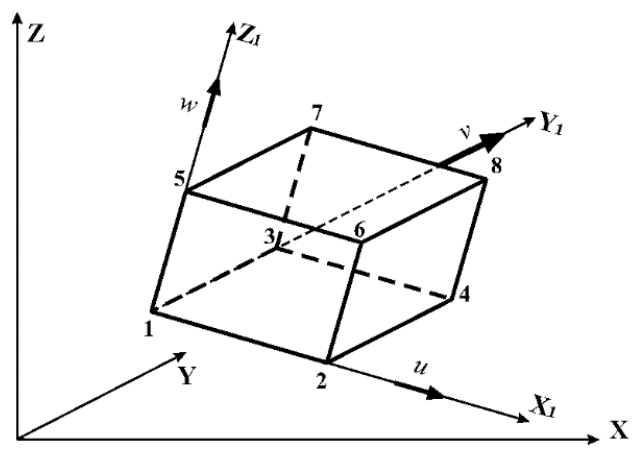

Fig. 1. FE volume in the form of a parallelepiped for modeling concrete.

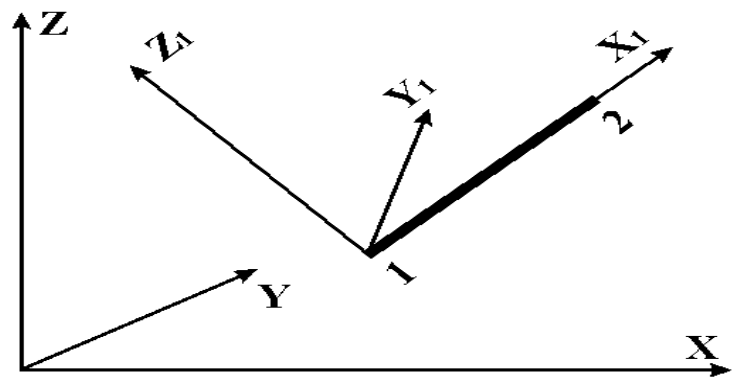

Fig. 2. FE rod for modeling steel reinforcement.

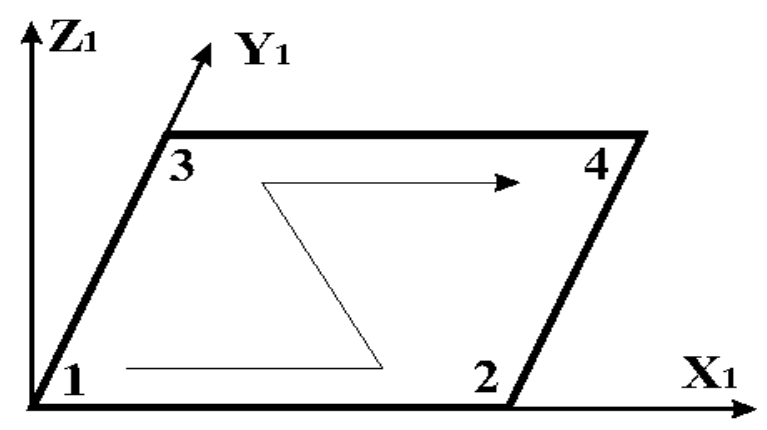

Fig. 3. FE shell for modeling composite stirrups. 


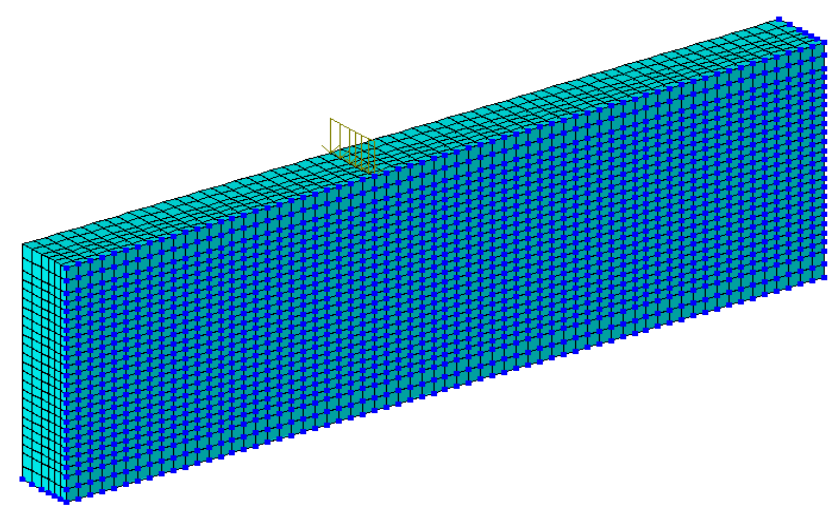

Fig. 4. Design scheme in SP LIRA SAPR.

\section{Materials and methods}

An exponential law was chosen as the deformation law for concrete, steel and carbon fiber (Fig. 5), which includes the following parameters:

$E_{0}^{+}$-initial tensile modulus of material;

$E_{0}^{-}$-initial modulus of the material elasticity in compression;

$\sigma_{\mathrm{p}}^{+}$-ultimate tensile strength;

$\sigma_{\mathrm{p}}^{-}$-ultimate compression resistance;

$\varepsilon_{\mathrm{p}}^{+}$-ultimate deformation of a material under tension;

$\varepsilon_{\mathrm{p}}^{-}$-ultimate deformation of a material in compression.

The exponential law equation for positive voltages is written as:

$$
\sigma=\sigma_{u l t}^{+}\left(1-\exp \left(-\frac{E_{0}^{+} \varepsilon}{\sigma_{\text {пр }}^{+}}\right)\right)
$$

(1)

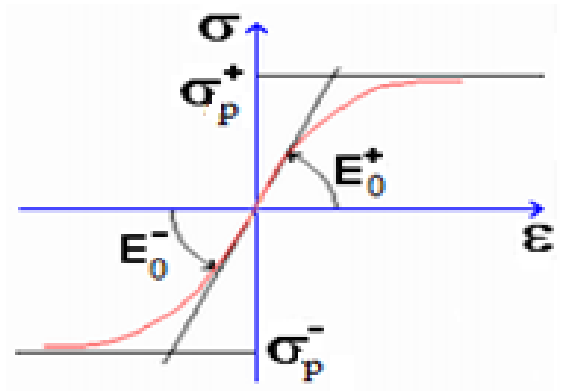

Fig. 5. Exponential law of material deformation.

The following values of physical and mechanical characteristics were taken for concrete: $E_{0}^{+}==E_{0}^{-}=3.25 \cdot 10^{4} \mathrm{MPa}, \sigma_{\mathrm{p}}^{+}=1.8 \mathrm{MPa}, \sigma_{\mathrm{p}}^{-}=22 \mathrm{MPa}$. For steel, these values were $E_{0}^{+}=E_{0}^{-}==2 \cdot 10^{5} \mathrm{MPa}, \sigma_{\mathrm{p}}^{+}=\sigma_{\mathrm{p}}^{-}=500 \mathrm{MPa}$, and for carbon fiber $-E_{0}^{+}=$ $E_{0}^{-}=2.3 \cdot 10^{5} \mathrm{MPa}, \sigma_{\mathrm{p}}^{+}==\sigma_{\mathrm{p}}^{-}=3066 \mathrm{MPa}$.

In addition to the exponential law, a piecewise linear deformation law was used for concrete. As an equation describing the relationship between stresses and strains in a uniaxial stress state, the Sarg in formula presented in the Eurocode was specified, which has the form: 


$$
\frac{\sigma}{R}=\frac{k \eta-\eta^{2}}{1+(k-2) \eta}
$$

where $R$ defines ultimate stress for concrete in compression (tensile), $\eta=\frac{\varepsilon}{\varepsilon_{R}} ; \varepsilon_{R}$ denotes deformation of concrete at $\sigma=R ; k$ is acurvature factor $\sigma-\varepsilon ; k=1 / \lambda_{R}$, where $\lambda_{R}$ is a coefficient of change of the secant modulus (coefficient of elasticity of concrete) at the top of the diagram

$\sigma-\varepsilon$.

The quantities $R, \lambda_{R}$ and $\varepsilon_{R}$ are related to the initial modulus of elasticity of concrete $E_{0}$ with the next addiction:

$$
R=E_{0} \varepsilon_{R} \lambda_{R}
$$

Deformation $\varepsilon_{R}$ was taken equal to $2 \cdot 10^{-3}$ for the compressed zone and $10^{-4}$ for the

\begin{tabular}{|c|c|c|c|}
\hline Strain & Stress & ^ & Draw \\
\hline-0.0016 & -16.9608 & & \multirow{8}{*}{$\sigma$} \\
\hline-0.0014 & -16.9583 & & \\
\hline-0.0012 & -16.5986 & & \\
\hline-0.001 & .15 .8033 & & \\
\hline-0.0008 & .14 .469 & & \\
\hline-0.0006 & -12.4568 & & \\
\hline-0.0004 & .9 .5756 & & \\
\hline-0.0002 & .5 .551 & $\checkmark$ & \\
\hline
\end{tabular}
stretched zone. The curve $\sigma-\varepsilon$ was built according to 20 points (Fig. 6).

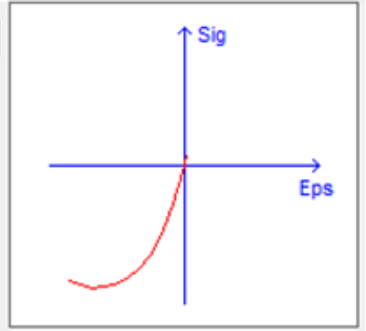

Fig. 6. Curve $\sigma-\varepsilon$ when specifying the law of deformation of concrete with Sarg in formula.

A step-iteration method with an automatic step selection for physically nonlinear problems was chosen as the method for solving a nonlinear problem. Maximum number of iterations - 400.

Calculation results at Poisson's ratio of concrete $v=0.3,0.4$ and 0.495 , and their comparison with the experimental data are presented in Table 1.

Poisson's ratio for steel and CFRP was taken equal to 0.3. The orthotropy of the composite material was not taken into account, since the software package LIRA does not allow to simultaneously take into account physical nonlinearity and anisotropy, but this could hardly have a significant effect on the results, since carbon fiber clamps mainly work in tension in one direction. For the Poisson's ratio of concrete, a calculation with values ranging from $v=0.2$ till $v=0.495$ was performed. Value $v=0.2$ is contained in the design standards for reinforced concrete structures, however, in the limiting state due to cracking, the Poisson's ratio of concrete can reach 0.5 or more. It is impossible to set Poisson's ratio greater or equal to 0.5 in the LIRA software package, so a value close to 0.5 was chosen as the upper bound.

\section{Results and Discussions}

As a result of calculations, it was found that the fracture pattern and the value of the limiting transverse force significantly depend on the selected value of the Poisson's ratio of concrete.

Finite element analysis was performed for three reinforcement options: closed composite clamps, U-clamps, and clamps on the lateral sides of the beam. In addition, a reference beam without reinforcement was calculated. When calculating with the value $v=$ 0.2 the value of the breaking load turned out to be the same both for the three reinforcement 
options and for the reference beam and amounted to $97.9 \mathrm{kN}$. The scheme of destruction, as can be seen from Fig. 7 - 9, for these cases also differs insignificantly. There is a slight decrease in stresses in the concrete, in the immediate vicinity of the CFRP clamps. As in the experiment, the presented figures show an oblique crack going from the support to the force application point, but the fracture is not accompanied by rupture of the composite clamps. The maximum tensile stresses in them for the three reinforcement options at the moment of fracture were $999 \mathrm{MPa}$.

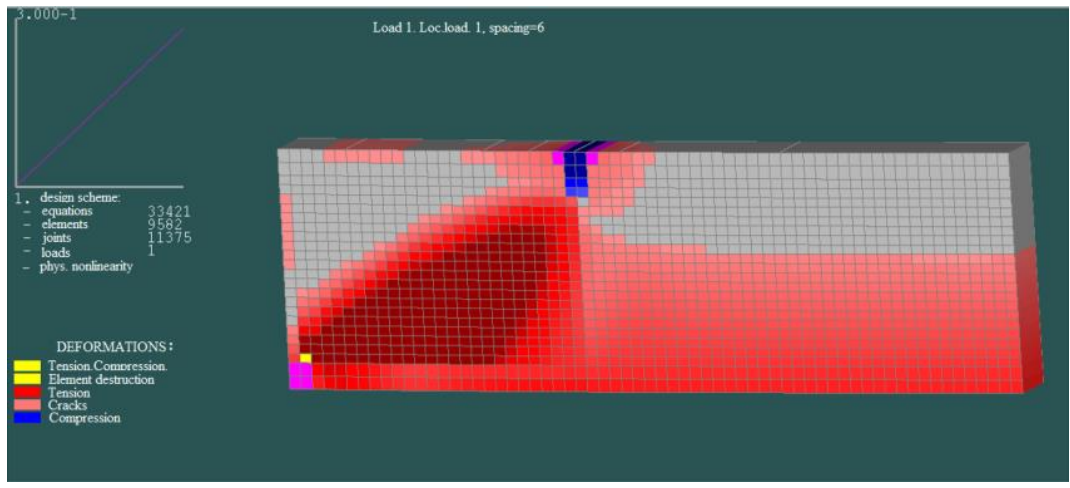

Fig. 7. Destruction scheme of a reference beam without reinforcement: $a$ - damaged elements at a load of $33 \%$ of the destructive one, $b$ - the same at a load of $67 \%$ of the destructive one, $c$ - at the moment of the structure destruction.

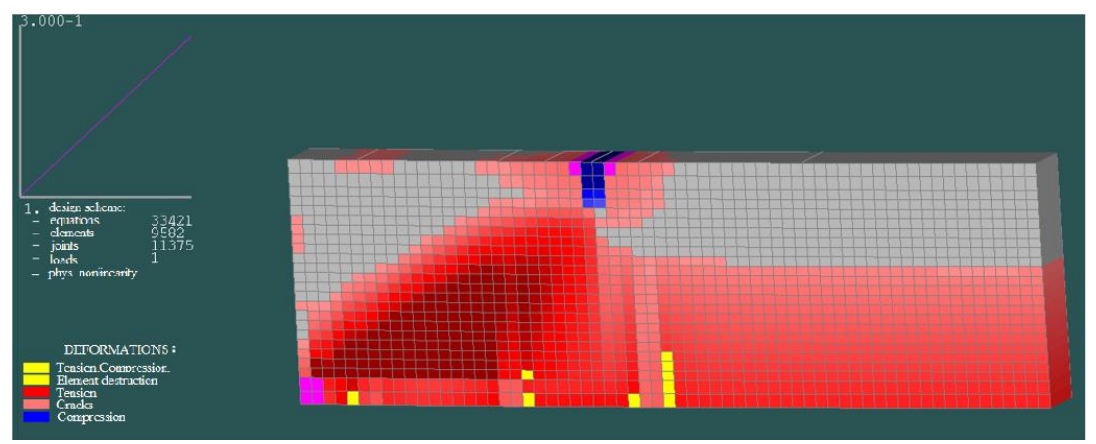

Fig. 8. Destruction diagram of the beams with closed and U-shaped clamps: $a$ - damaged elements at a load of $33 \%$ of the destructive, $b$ - the same at a load of $67 \%$ of the destructive, $c$ - at the moment of the structure destruction.

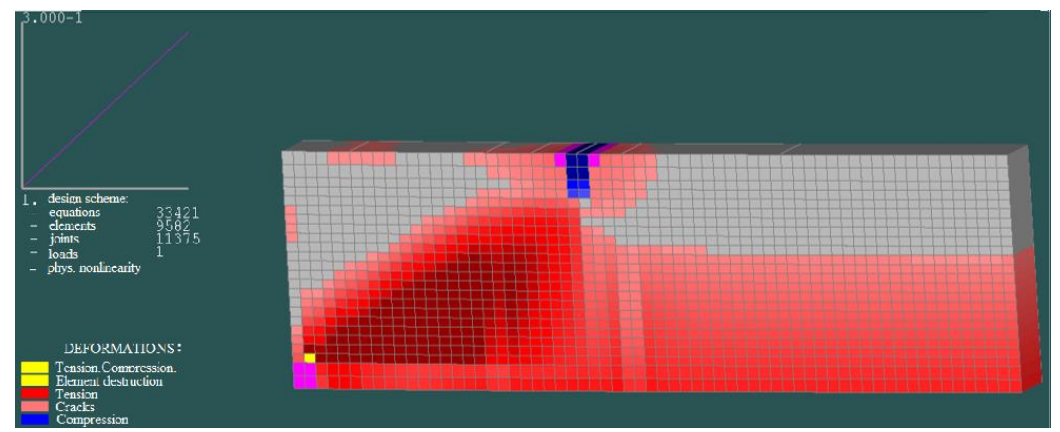

Fig. 9. Destruction diagram of a beam with double-sided composite clamps: $a$ - damaged elements at a load of $33 \%$ of the destructive one, $b$ - the same at a load of $67 \%$ of the destructive one, $c$ - at the moment of the structure destruction. 
Table 1. Comparison of the experimental and theoretical obtained in SP LIRA, strength of inclined sections of beams, without initial cracks.

\begin{tabular}{|c|c|c|c|c|c|}
\hline \multirow{2}{*}{} & \multicolumn{4}{|c|}{ Breaking load $Q_{u l t},[k N]$} \\
\cline { 2 - 6 } & $\begin{array}{c}\text { Reference } \\
\text { beam }\end{array}$ & $\begin{array}{c}\text { Closed } \\
\text { clamps }\end{array}$ & U-clamps & $\begin{array}{c}\text { Double- } \\
\text { sided clamps }\end{array}$ \\
\hline \multirow{2}{*}{ Experiment } & 102.75 & - & 128.48 & 119.75 \\
\hline \multirow{2}{*}{$\begin{array}{c}\text { SP } \\
\text { LIRA }\end{array}$} & $v=0.3$ & 97.95 & 123.05 & 123.05 & 123.08 \\
\cline { 2 - 6 } & $v=0.4$ & 97.90 & 122.75 & 122.68 & 122.73 \\
\cline { 2 - 6 } & $v=0.495$ & 92.93 & 108.10 & 108.10 & 92.91 \\
\hline
\end{tabular}

Table 1 shows that when calculating in SP LIRA, the results closest to the experimental data were obtained for reference beams and beams with $U$-shaped clamps at $\nu=0.3$, and for double-sided clamps - with $v=0.4$. For the reference beam, the discrepancy was $4.7 \%$, when reinforced with U-clamps $-4.2 \%$, and when reinforced with double-sided composite clamps - only $2.5 \%$. In this case, the values of the breaking load along the inclined section at $v=0.3$ differ little from the values $Q_{u l t}$ at $v=0.4$.

It should be noted that increasing the Poisson's ratio for all finite elements is a simplified approach and does not fully reflect the specifics of concrete operation in the limiting state. Poisson's ratio is increased not in all finite elements, but only in fracture zones. Thus, there is a need to improve the models of concrete destruction included in the design complexes so that they allow taking into account the change in Poisson's ratio when approaching the limiting state.

\section{Conclusion}

The difference in the results when specifying the concrete deformation curve by the exponential and piecewise linear law turned out to be insignificant, i.e., the breaking load magnitude is mainly influenced not by the diagram nature, but by the limiting values of stresses and strains.

\section{References}

1. D.R. Mailyan, P.P. Polskoy, H. Mervat, K.V. Kurgin, Don Engineering Herald 4 (2013).

2. M. Hishmah, D.R. Mailyan, P.P. Polskoy, A.M. Blyagoz, New technologies 4 (2012).

3. P.P. Polskoy, M. Khishmakh, A. Mikhub, Scientific Review 6 (2012).

4. P.P. Polskoy, D.R. Mailyan, Don Engineering Herald 2 (2013).

5. P.P. Polskoy, D.R. Mailyan, D.A. Dedukh, S.V. Georgiev, Global journal of Pure and Applied mathematic 2 (2016).

6. P.P. Polskoy, MSF 931 (2013).

7. P.P. Polskoy S.V. Georgiev, V.A. Muradyan, A.A. Shilov, MATEC Web of Conferences 196, 02026 (2018). 
8. P.P. Polskoy, S.V.Georgiyev, Don Engineering Herald 4 (2013).

9. P.P. Polskoy, S.V.Georgiyev, Scientific Review 10 (2014).

10. P.P. Polskoy, D.R. Mailyan, A.A. Shilov, Z.A. Meretukov, Newtechnologies 4, 35 (2015).

11. P.P. Polskoy, D.R. Mailyan, A.A. Shilov, Z.A. Meretukov, Newtechnologies 4, 44 (2015).

12. P.P. Polskoy, D.R. Mailyan, A.A. Shilov, K.V. Shevlyakov, Don Engineering Herald 4 (2016)

13. P.P. Polskoy, D.R. Mailyan, A.A. Shilov, P.A. Shilov, E3S Web of Conferences 110, 01053 (2019).

14. P.P. Polskoy, D.R. Mailyan, A.A. Shilov, MSF 974, 633 (2019). 